\title{
Examining the nutritional quality of the product portfolios of major packaged food and beverage companies in Canada
}

\author{
Laura Vergeer $^{1}$, Mavra Ahmed ${ }^{1}$, Lana Vanderlee ${ }^{1,2}$ and Mary L'Abbé ${ }^{1}$ \\ ${ }^{1}$ Department of Nutritional Sciences, University of Toronto, Toronto, Canada and \\ ${ }^{2}$ School of Public Health and Health Systems, University of Waterloo, Waterloo, Canada
}

\begin{abstract}
Canada's food supply is abundant in energy-dense products containing excess amounts of sodium, saturated fat and free sugars, increasing Canadians' risk of developing obesity and non-communicable diseases. Food companies shape the food supply through their control over the formulation of their products; however, no studies have examined the healthfulness of products offered by different companies in Canada. This study aimed to assess and compare the nutritional quality of the product portfolios of major packaged food and beverage companies in Canada. Twenty-two top food companies were selected for study, representing a combined $50 \%$ and $73 \%$ of Canadian packaged food and beverage sales in 2018, respectively. This included 18 multinational companies, 2 Canadian manufacturers and 2 retailers with private-label brands. Nutritional information for products was sourced from the University of Toronto Food Label Information Program 2017 database. The nutritional quality of all products offered by the sampled companies that were included in the database $(n=8,211)$ were evaluated using the Health Star Rating (HSR) system, with HSRs ranging from 0.5 (less healthy) to 5 (healthier). Descriptive analyses and analysis of variance with post-hoc tests examined the HSRs of each company's products overall and by food category $(\mathrm{n}=24)$. Mean HSRs of companies' overall product portfolios ranged from 1.8 to $3.7\left(\mu_{\overline{\mathrm{x}}}=2.7\right.$, $\left.\sigma_{\overline{\mathrm{x}}}=0.5\right)$ and differed significantly between companies $(p<0.001)$. Mean HSRs differed between companies for all food categories except eggs $(p=0.5)$, seafood $(p=0.2)$, legumes $(p=0.1)$, nuts and seeds $(p=0.4)$, and vegetables $(p=0.08)$. Variation in mean HSRs of products offered by different companies was greatest for beverages (range $\left.=1.3-5.0, \mu_{\bar{x}}=2.0, \sigma_{\bar{x}}=1.0\right)$, fats/oils $($ range $=$ $\left.0.7-4.4, \mu_{\bar{x}}=3.6, \sigma_{\bar{x}}=1.6\right)$, fruit/fruit juices (range $\left.=0.8-4.0, \mu_{\bar{x}}=2.6, \sigma_{\bar{x}}=0.9\right)$, and sauces/dips/gravies/condiments $(\mathrm{range}=0.5-$ $3.4, \mu_{\bar{x}}=2.3, \sigma_{\bar{x}}=1.0$ ). These findings demonstrate that the nutritional quality of products offered by leading food manufacturers in Canada varies significantly overall and by food category, with many of these products considered less healthy according to the HSR system. Differences between companies may reflect the nature of their products; for example, products offered by dairy companies were healthier than those of confectionary and soft drink manufacturers, on average. Variation in nutritional quality within food categories illustrates the need and potential for many companies to improve the healthfulness of their products. By identifying companies that offer less healthy products compared with others in Canada, this study may prompt reformulation.
\end{abstract}

\section{Conflict of Interest}

The first author has completed the disclosure form and declares: Mavra Ahmed is a Mitacs Elevate Postdoctoral Fellow at the University of Toronto and is funded jointly by Mitacs and the Nestlé Research Center. Mary L'Abbé reports grants from the Retail Council of Canada, Program for Food Safety, Nutrition and Regulatory Affairs at the University of Toronto (with partial funding from Nestlé Canada), and Dairy Farmers of Canada; these grants are unrelated to the submitted work. None of these companies/organizations had any involvement in the present research. The other authors have no conflicts of interest to declare. 